\title{
Anatomia da madeira e potencial de produção de celulose e papel de quatro espécies jovens de Eucalyptus L'Hér.
}

\author{
Talita Baldin ${ }^{1}$, José Newton Cardoso Marchiori ${ }^{1}$, Silvana Nisgoski ${ }^{2}$, Maiara Talgatti ${ }^{1 \star}$, \\ Luciano Denardi ${ }^{1}$ \\ ${ }^{1}$ Departamento de Ciências Florestais (PPGEF), Centro de Ciências Rurais, Universidade Federal de Santa Maria, Santa \\ Maria-RS, Brasil. \\ ${ }^{2}$ Departamento de Ciências Florestais (PPGEF), Setor de Ciências Agrárias, Universidade Federal do Paraná, Curitiba-PR, \\ Brasil.
}

\begin{abstract}
RESUMO No Brasil, a matéria-prima utilizada na produção de celulose e papel é proveniente do gênero Eucalyptus L'Hér., obtida em florestas de curta rotação, produtores de madeira com alto rendimento em celulose de fibra curta, e excelentes propriedades anatômicas, químicas e físicas. Objetiva-se então, avaliar a qualidade da madeira de indivíduos jovens de quatro espécies de Eucalyptus, por meio da anatomia do lenho, e obter maiores informações sobre o potencial das mesmas para produção de celulose e papel. Foram retirados discos na altura do peito de árvores com cinco anos de idade de Eucalyptus benthamii, E. dunnii, E. grandis e E. saligna. Os índices de qualidade da madeira para celulose e papel (Índice de Runkel, Fração de Parede, Flexibilidade, Rigidez e Fator de Luce) foram calculados a partir dos valores médios da morfologia das fibras, obtidos do alburno. Embora anatomicamente semelhantes, poros em agrupamento de três ou mais unidades, em Eucalyptus saligna, e a presença de parênquima paratraqueal aliforme, em Eucalyptus dunnii, permitem diferenciação das mesmas. Os índices de qualidade mostram, que Eucalyptus benthamii, E. grandis e E. saligna, podem ser classificadas como boas para papéis de alta resistência mecânica, ao passo que E. dunnii indica-se para papéis do tipo absorventes.
\end{abstract}

Palavras-chave: Variação radial; Índices qualitativos da madeira; Comprimento de fibras; Madeira juvenil.

\section{Anatomy of wood and potential of cellulose and paper production of four young species of Eucalyptus L'Her.}

\begin{abstract}
In Brazil, the raw material used for pulp and paper production comes from the genus Eucalyptus L'Hér, high yields of hardwood pulp with excellent anatomical, chemical and physical properties of woods. In this sense, the objective of this paper is to evaluate through anatomy, the young wood of four Eucalyptus species, in order to obtain more information about its potential for pulp and paper production. Breast hight discs, from four 5-year-old Eucalyptus benthamii, E. dunnii, E. grandis and E. saligna were used for anatomical studies. The wood quality values for pulp and paper production (Runkel ratio, Wall proportion, Flexibility coefficient, Slenderness ratio, Luce's factor) were defined based on mean values of sapwood fiber morphology. Although anatomically similar, pores in three or more units, in Eucalyptus saligna, and paratracheal aliform parenchyma, in E. dunnii, allows their differentiation. The quality indexes show that Eucalyptus benthamii, E. grandis and E. saligna produce nice woods for high mechanical resistance paper, while E. dunnii can be indicated for absorbent type papers.
\end{abstract}

Keywords: Radial variation; Qualitative indices of wood; Fiber length; Juvenile wood.

\section{Introdução}

Desde os anos 60, a produção de celulose e papel no Brasil tem crescido em taxas superiores às vislumbradas pelos demais produtores no mundo. Atualmente, o país é o quarto produtor mundial de celulose, atrás apenas de Estados Unidos, Canadá e China, e ocupa a nona posição na produção de papel. Nesse ritmo, considerando as últimas seis décadas, o Brasil apresenta um crescimento médio de produção anual de 7,5\% na celulose, e de quase $6 \%$ na fabricação de papel, muito acima da média histórica mundial de 2,2\% na celulose e de 3,7\% no papel (IBÁ, 2016). 
Os dados demonstram a potencialidade do país para a modernização e ampliação do parque fabril.

No tocante à matéria-prima utilizada para a produção de celulose e papel, há destaque para as espécies do gênero $E u$ calyptus L'Hér., face as florestas de curta rotação, alto rendimento em celulose de fibra curta e excelentes propriedades físicas e químicas da madeira, conforme demonstrado por Gomide et al. (2005). Embora seja tradição no Brasil o uso de Eucalyptus grandis, E. saligna e do hibrido E. grandis $\mathrm{x}$ urophylla para a produção de celulose de mercado, torna-se necessário o estudo de novas espécies, como o Eucalyptus benthamii, que tem se sobressaído pela resistência às condições de geada que ocorrem em ambientes subtropicais (SILVA et al., 2012).

Em programas de melhoramento genético no setor florestal, voltados à produção de celulose e papel, os principais fatores considerados para a seleção das espécies envolvem a taxa de crescimento das árvores, representada pelo incremento médio anual (REIS et al., 2015) e a densidade básica da madeira (RAYMOND; MUNERI, 2001). Sabe-se, contudo, que outros caracteres também devem ser avaliados, como a composição química e anatômica da madeira, bem como o processo e a condição de formação da pasta.

A qualidade do papel é diretamente influenciada pelas características morfológicas das fibras e pela fração das mesmas na polpa (GOMIDE et al., 2005). Madeiras com fibras de paredes mais espessas, por exemplo, produzem um papel mais poroso e opaco, conferindo melhor capacidade de impressão. Já as fibras de paredes mais finas, contrariamente, determinam a formação de papéis mais densos e com elevada resistência a ruptura e a tensão (WIEDENHOEFT; MILLER, 2005). Existe, ainda, uma estreita relação entre o comprimento das fibras e a produção do papel: em geral, fibras curtas contribuem para uma boa formação de folha, ao passo que as mais longas favorecem a resistência ao rasgo (GOMIDE et al., 2005).

A presença de elementos vasculares favorece a penetração e impregnação dos cavacos pelo licor de cozimento kraft. Entretanto, não é desejável que a madeira apresente alto teor dessas células, pois implica diretamente em menor densidade, o que proporciona menor produtividade na fábrica e maior consumo específico de material (ALVES et al., 2011).

Embora as espécies de eucalipto sejam amplamente pesquisadas, poucos são os trabalhos que investigam profundamente esse material e relacionam caracteres intrínsecos da madeira com a produção de celulose e papel. Nesse sentido, a pesquisa teve como objetivo avaliar a qualidade tecnológica da madeira de quatro espécies jovens de Eucalyptus, por meio da anatomia do lenho, visando obter maiores informações sobre o seu potencial para esse segmento de mercado.

\section{Material e Métodos}

\section{Caracterização geral do experimento}

A madeira para os estudos anatômicos é proveniente de árvores de Eucalyptus benthamii Maiden \& Cambage, Eucalyptus dunnii Maiden, Eucalyptus grandis W. Hill e Eucalyptus saligna Sm., coletadas em plantio experimental de propriedade da empresa CMPC Celulose Riograndense, localizado no município de Encruzilhada do Sul, Rio Grande do Sul, sob as coordenadas S -30.65268 e O -52.46839 .

O corte das árvores foi realizado em março de 2016, quando essas tinham 5 anos de idade, sendo selecionados, aleatoriamente, 3 indivíduos por espécie, e retirado discos de madeira na altura de $1,3 \mathrm{~m}$ (diâmetro a altura do peito), totalizando 12 amostras de madeira. Os dados dendrométricos de altura total (ht), diâmetro a altura do peito (dap) e volume com casca (vcc), mensurados antes do abate das árvores, estão sumarizados na Tabela 1 . 
Tabela 1. Árvores amostradas no estudo com os respectivos dados dendrométricos.

Table 1. Trees samples in the study with respective dendrometric data.

\begin{tabular}{cccc}
\hline Espécie & ht $\mathbf{( c m})$ & dap $(\mathbf{c m})$ & vcc $\left(\mathbf{c m}^{\mathbf{3}}\right)$ \\
\hline Árvore 1 - Eucalyptus benthamii & 19,90 & 15,95 & 0,1789 \\
Árvore 2 - Eucalyptus benthamii & 14,50 & 9,36 & 0,0449 \\
Árvore 3 - Eucalyptus benthamii & 19,70 & 17,70 & 0,2181 \\
Árvore 1 - Eucalyptus dunnii & 10,44 & 15,0 & 0,0578 \\
Árvore 2 - Eucalyptus dunnii & 18,40 & 19,70 & 0,2357 \\
Árvore 3 - Eucalyptus dunnii & 16,11 & 18,30 & 0,1678 \\
Árvore 1 - Eucalyptus grandis & 18,21 & 21,40 & 0,2507 \\
Árvore 2 - Eucalyptus grandis & 19,03 & 19,70 & 0,2523 \\
Árvore 3 - Eucalyptus grandis & 17,40 & 11,30 & 0,0785 \\
Árvore 1 - Eucalyptus saligna & 20,90 & 17,79 & 0,2339 \\
Árvore 2 - Eucalyptus saligna & 16,30 & 10,60 & 0,0647 \\
Árvore 3 - Eucalyptus saligna & 12,30 & 8,44 & 0,0309 \\
Total: 12 árvores & & &
\end{tabular}

Onde: ht: altura total, dap: Diâmetro a altura do peito, vcc: Volume com casca.

Where: ht: total height, dap: Diameter at breast height, vcc: Volume with bark.

\section{Descrição anatômica das madeiras}

De cada amostra de madeira (disco) foram retirados corpos de prova na direção medula-casca (cerne, cerne periférico e alburno) e confeccionadas lâminas de macerado para determinação da variação radial de fibras. Também, foram extraídos mais três corpos de prova, com dimensões $3 \times 3 \times 3 \mathrm{~cm}$, na região do alburno, devidamente orientados, para obtenção de cortes histológicos nos planos transversal (X), longitudinal radial (R) e longitudinal tangencial (T) (Figura 1).

O preparo de lâminas de macerado seguiu o método de Jeffrey (FREUND, 1970) e a montagem das lâminas histológicas seguiu a técnica padrão, recomendada por Burger e Richter (1991), ambos realizados no laboratório de anatomia da madeira da Universidade Federal de Santa Maria, Rio Grande do Sul.

A descrição microscópica seguiu basicamente as recomendações do IAWA Committee (1989), com exceção da percentagem dos distintos tecidos, da altura e largura de raios (inclusive de seu corpo e margens, em número de células), bem como da espessura da parede de fibras e vasos, aspectos não contemplados na referida norma, que seguiram a metodologia utilizada por Marchiori (1982). A mensuração dos caracteres anatômicos da madeira foi realizada em microscópio binocular Carl Zeiss, modelo Standard 25, provido de ocular com escala graduada. As fotomicrografias foram tomadas em microscópio Nikon FXA, modelo E200, equipado com câmera digital, no laboratório de anatomia da madeira do Instituto Superior de Agronomia, Portugal.

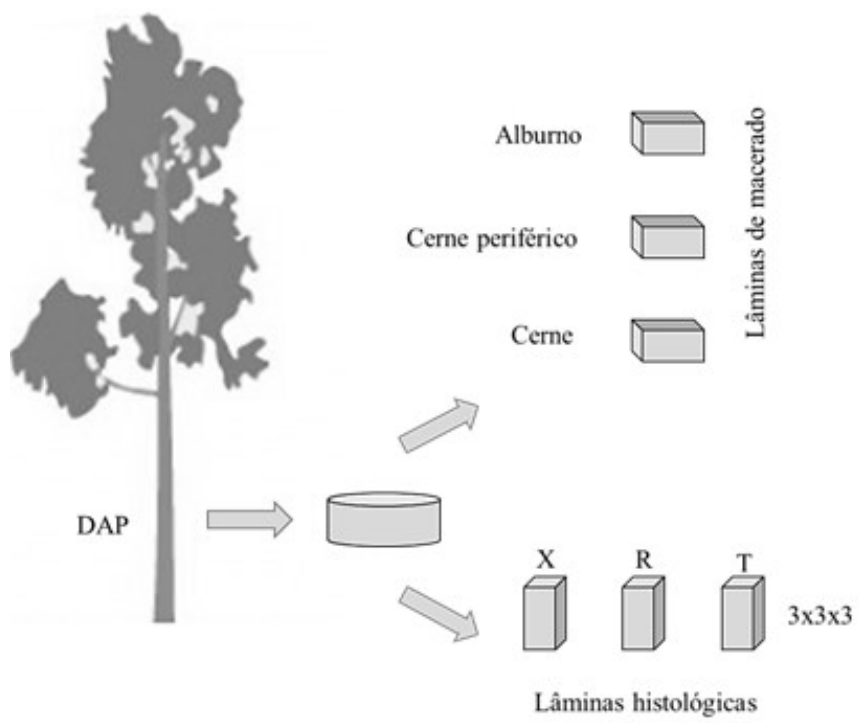

Figura 1. Processo de amostragem para confecção de lâminas anatômicas.

Figure 1. Sampling process for making anatomical slides. 


\section{Variação radial das dimensões das fibras $e$}

\section{índices qualitativos da madeira}

Para determinação da variação radial do comprimento, da largura e da espessura de fibra nas posições cerne, cerne periférico e alburno os seguintes caracteres anatômicos foram mensurados: comprimento (L), diâmetro ou largura total (Dt), diâmetro do lúmen (Dl) e espessura da parede (e) de 25 fibras por posição, totalizando 75 mensurações em cada árvore/amostra e 225 para cada espécie.

A partir dos valores médios obtidos da posição alburno, foram calculados os seguintes índices qualitativos, considerados importantes para produção de papel e relacionados às propriedades físico mecânicas do material produzido: coeficiente de flexibilidade $(\mathrm{CF})$, obtido por $\mathrm{CF}=(\mathrm{Dl} / \mathrm{Dt}) \times 100$ (MILANEZ; FOELKEL, 1981); fração de parede (FP), determinado pela equação $\mathrm{FP}=(2 \mathrm{e}) / \mathrm{Dt} \times 100($ FOELKEL et al., 1978); e índice de Runkel (IR), obtido por IR = 2e/Dl (RUNKEL, 1952), coeficiente de rigidez (CR), determinado por CR $=\mathrm{L} / \mathrm{Dt}$ (OGBONNAYA et al., 1997; SAIKIA et al., 1997) e fator de forma de Luce $=\left(\mathrm{Dt}^{2}-\mathrm{Dl}^{2}\right) /\left(\mathrm{Dt}^{2}+\mathrm{Dl}^{2}\right)(\mathrm{LUCE}, 1970)$.

\section{Análise estatística}

As características anatômicas quantitativas foram analisadas em delineamento inteiramente casualizado - DIC, utilizando o programa estatístico Statistica 8.0. Quando houve a constatação de diferença significativa entre os tratamentos (espécies) pelo teste $\mathrm{F}(\mathrm{p} \leq 0,05)$, aplicou-se o teste Tukey ( $\mathrm{p}$ $\leq 0,05)$ para a comparação entre as médias de cada parâmetro avaliado. Para a variação radial do comprimento, da largura e da espessura de fibra, obteve-se os valores médios, desvio padrão e coeficiente de variação. Os índices qualitativos (coeficiente de flexibilidade, fração parede, índice de Runkel, coeficiente de rigidez e fator de luce) foram expressos em mínimo, médio, máximo e desvio padrão.

\section{Resultados e Discussão}

\section{Análise qualitativa da madeira}

As espécies do gênero Eucalyptus investigadas caracterizam-se por apresentar estrutura anatômica semelhante do ponto de vista qualitativo, tornando difícil a tarefa de distingui-las, de forma segura, com base unicamente nesse critério (Figura 2)

As espécies de Eucalyptus reúnem características típicas de Myrtaceae, conforme pode ser verificado na bibliografia especializada: Dadswell e Burnell (1932); Record e Hess (1949); Metcalfe e Chalk (1983); Détienne e Jacquet (1983); Vliet e Baas (1984) e Santos et al. (2015). É o caso da porosidade difusa, das placas de perfuração simples e das pontoações intervasculares alternas. Os eucaliptos evidenciam uma estrutura anatômica evoluída, com elementos vasculares curtos, parênquima axial paratraqueal e fibras libriformes.

As madeiras pesquisadas constituem-se, majoritariamente por fibras (45\%), raios (20\%) e, em menores porcentagens, por parênquima axial (17\%), elementos vasculares (17\%) e traqueídes vasculares (1\%), atestando que, além de todos os aspectos silviculturais que envolvem o eucalipto, a proporção dos tecidos e a quantidade de fibras na madeira é fator determinante no emprego desse gênero na indústria de celulose e papel. Foelkel e Barrichelo (1975) e Barrichelo e Brito (1976), indicam, de maneira geral, para a produção de celulose com eucalipto, porcentagem de fibras (fibras libriformes + fibrotraqueídeos) de cerca de $65 \%$, vasos de $17 \%$, e tecido parenquimatoso $18 \%$.

Os anéis de crescimento são indistintos em Eucalyptus benthammii e E. dunnii, enquanto em E. grandis e E. saligna são marcados, fracamente, por fibras radialmente estreitas no lenho tardio. Embora todas as espécies tenham apresentado poros predominantemente solitários e raros múltiplos radiais com a média de 2 unidades, E. saligna distinguiu-se, por mos- 


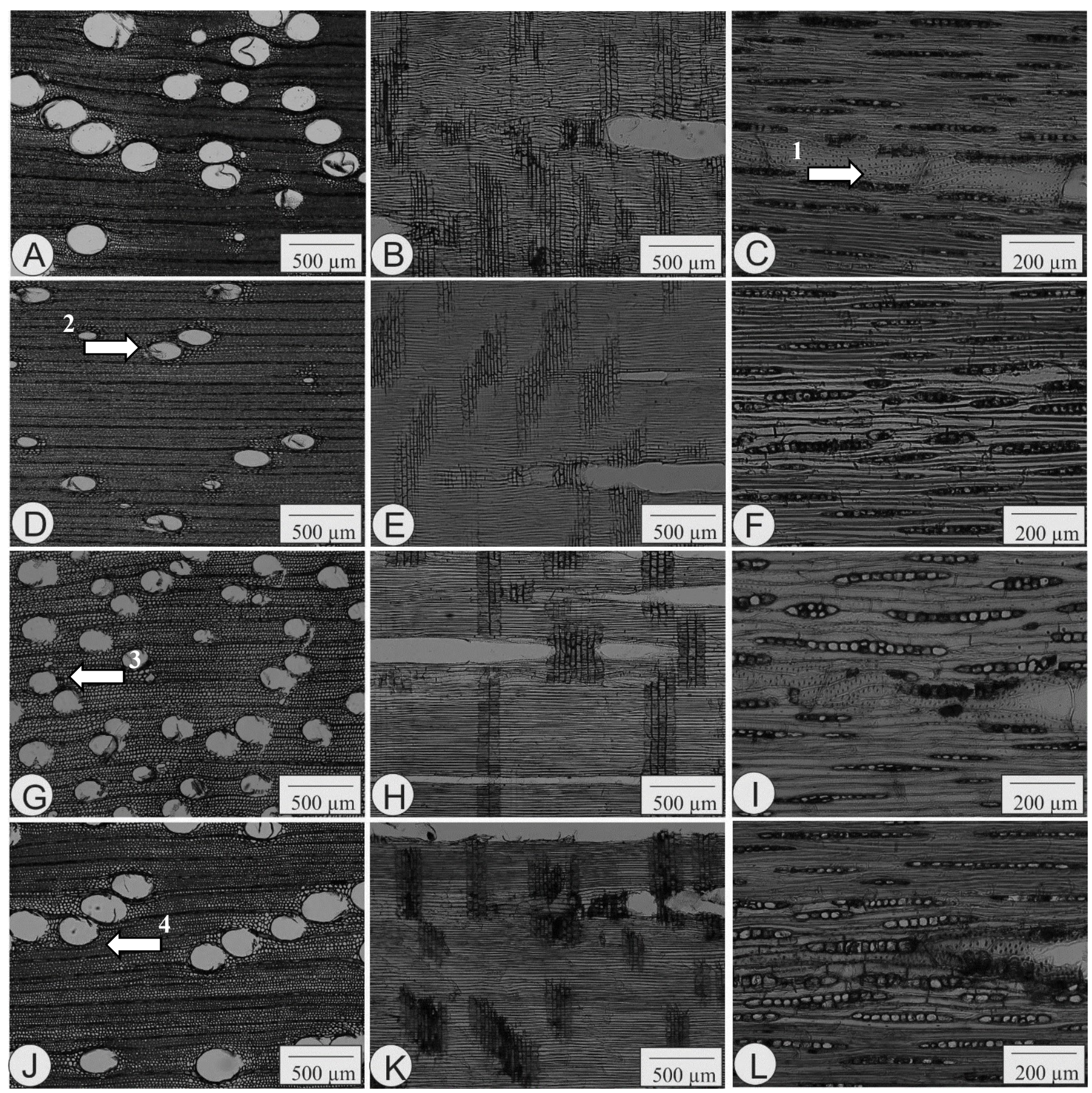

Figura 2. Eucalyptus benthamii em seção transversal (A), radial (B) e tangencial (C). A seta 1 aponta para a presença de traqueídes vasculares. Eucalyptus dunnii em seção transversal (D), radial (E) e tangencial (F) evidenciando parênquima paratraqueal aliforme (seta 2). Eucalyptus grandis em seção transversal (G), radial (H) e tangencial (I). A seta 3 evidencia poros solitários. Seção transversal (J), radial (K) e tangencial (L) em Eucalyptus saligna. Poros agrupados em três unidade (seta 4).

Figure 2. Cross (A) Radial (B) Tangential section in Eucalyptus benthamii. The arrow 1 shows vascular tracheids. Cross (A) Radial (B) Tangential section in Eucalyptus dunnii show axial paratracheal aliforme parenchima (arrow 2). Cross (A) Radial (B) Tangential section in Eucalyptus grandis. The arrow 3 shows solitary pores. Cross (A) Radial (B) Tangential section in Eucalyptus saligna. Presence of radial multiple grouping (arrow 4).

trar poros agrupados em 3 até 8 unidades (Figura 2).

As espécies apresentam porosidade difusa, não uniforme; os poros são numerosos (entre $60-150$ poros $/ \mathrm{mm}^{2}$ ), de secção arredondada a oval, em arranjo diagonal. Placas de perfuração simples e oblíquas. Pontoações raio-vasculares grandes, ovais, com aréola reduzida, sem arranjo definido e, aparentemente, com maior incidência na porção terminal dos raios. 
Pontoações parênquimo-vasculares semelhantes às intervasculares. Traqueídes vasculares presentes em todas as espécies.

A presença de traqueídes vasculares em Eucalyptus benthamii (Figura 2C) aparece como novidade na descrição anatômica da espécie e não foi mencionada anteriormente em estudos de Nisgoski et al. (1998) e Alves et al. (2011). Igualmente em E. dunnii e E. saligna, Gonçalves et al. (2014) não referem a ocorrência do elemento celular.

Poros em arranjo diagonal é característica constante em madeiras de Eucalyptus (WILKES, 1988; NISGOSKI et al., 1998; GONÇALVES et al., 2014). De nove espécies do gênero estudadas por Pirralho et al. (2014), apenas uma delas, Eucalyptus maculata Hook., apresenta arranjo radial de poros.

Parênquima axial raramente apotraqueal, com predomínio do tipo paratraqueal vasicêntrico, confluente, e, menos frequente, unilateral, em Eucalyptus benthammii e E. saligna e paratraqueal aliforme em E. dunnii (Figura 2D). A faixa parenquimática, nas quatro espécies, envolve os poros com mais de 3 células de largura. Semelhantemente ao encontrado neste estudo, Pirralho et al. (2014) descrevem parênquima vasicêntrico em E. camaldulensis Dehnh., E. melliodora Schauer., E. propinqua H.Deane \& Maiden, E. sideroxylon Woolls, E. tereticornis Sm. e E. viminalis Labill. Nisgoski et al. (1998) mencionam, para $E$. benthamii, parênquima vasicêntrico e vasicêntrico confluente.

Os raios são frequentemente unisseriados (cerca de 80\%) e em menor ocorrência, bisseriados. Raios heterogêneos, com células procumbentes ao centro e uma fileira nas margens superior e inferior de células quadradas; raros homogêneos, de células procumbentes. Record e Hess (1949) e Metcalfe e Chalk (1983) descrevem para a família Myrtaceae, de maneira geral, raios nitidamente heterogêneos.

A constituição dos raios é bastante variável no gênero $E$ ucalyptus. Alzete (2009), analisando clones de E. grandis x E. urophyla, evidencia presença exclusiva de raios homogêneos, formados por células procumbentes. Pirralho et al. (2014) refere raios homogêneos e heterogêneos em E. camaldulensis, E. globulus Labill., E. ovata Labill., E. propinqua Labill. e E. tereticornis.

Em geral, nas quatro amostras descritas, há grande similaridade quanto à organização do tecido fibroso. As fibras são libriformes, não septadas, médias a curtas, com pontoações areoladas $(<3 \mu \mathrm{m})$, e abertura em fenda vertical, nas faces radiais e tangenciais da parede, como descrito por Pirralho et al. (2014) em noves espécies de Eucalyptus.

É de suma importância salientar que as espécies de $E u$ calyptus presentemente investigadas possuem idade de formação de madeira juvenil, e nesse tipo de tecido, as características anatômicas podem, ou não, estar completamente formadas.

\section{Análise quantitativa da madeira}

Os valores médios encontrados para a maioria das características anatômicas quantitativas das espécies presentemente investigadas (Tabela 2) corroboram os relatados na literatura, em análises feitas com eucaliptos em condições e idades semelhantes (PIRRALHO et al., 2014; OLIVEIRA et al., 2012). As espécies podem ser discriminadas com base em seus dados quantitativos do lenho.

Elementos vasculares de maior comprimento e pontoações raio-vasculares de maior diâmetro são reportados em Eucalyptus grandis (530 $\mu \mathrm{m}$ e $11 \mu \mathrm{m}$, respectivamente). Para os elementos vasculares, todos os valores são superiores aos descritos por Pirralho et al. (2014) em 14 espécies de Eucalyptus com idades semelhantes, entre 164-276 $\mu \mathrm{m}$. Quanto à espessura da parede dos poros, E. benthamii apresenta paredes mais espessas $(9,3 \mu \mathrm{m})$.

Os raios multisseriados em Eucalyptus benthamii, embora apresentam as menores alturas, em números de células (10) e em micrômetros (217), são estatisticamente semelhantes a $E$. 
dunnii e E. grandis. Em E. saligna e E. dunnii são verificados, respectivamente, raios multisseriados com maiores alturas em número de células (13) e em micrometros (273). Para E. grandis, amostrados em duas diferentes regiões, Oliveira et al. (2012) refere altura média de 298,41 $\mu \mathrm{m}$, e 233,07 $\mu \mathrm{m}$, próximos a este estudo.

Quanto às séries de parênquima axial, Eucalyptus benthamii apresenta menores médias em altura $(283 \mu \mathrm{m})$ e largura $(17 \mu \mathrm{m})$, não diferindo, nesse caso, de E. dunnii $(15 \mu \mathrm{m})$. Parênquima axial com séries mais altas foram verificadas em $E$. dunnii e E. grandis.

As fibras são de comprimento médio, com $903 \mu \mathrm{m}$ em $E$. benthamii, e até $1078 \mu \mathrm{m}$ em E. saligna. As paredes fibrosas, delgadas a espessas, oscilam entre 3,2 $\mu \mathrm{m}$ (E. saligna) até 4,3 $\mu \mathrm{m}$ (E. dunnii). Oliveira et al. (2012) menciona valores quantitativos para as fibras de E. grandis muito semelhantes. Em E. benthamii, Nisgoski et al. (1998) verificaram comprimento de fibras na altura do DAP entre 800 e $850 \mu \mathrm{m}$. Em análise anatômica do caule de E. grandis, E. urophylla e E. grandis $\mathrm{x}$ urophylla, Brisola e Demarco (2011) caracterizam as espécies como tendo fibras curtas, estreitas a médias, com paredes delgadas a espessas. Fibras de paredes espessas, com $5 \mu \mathrm{m}$, são referidas por Pirralho et al. (2014) para E. globulus e E. maculata.

Cabe salientar que a determinação dos valores quantitativos das fibras é de crucial importância para se permitir previsões acerca do comportamento da polpa em condições operacionais de processo, sendo que as fibras se constituem no elemento anatômico mais abundante na madeira e seu comprimento, espessura e largura da parede afetam, diretamente, o rendimento e a qualidade do produto final.

Tabela 2. Valores médios das características anatômicas nas quatro espécies de eucalipto investigadas.

Table 2. Mean values of anatomical characteristics in the four eucalyptus species investigated.

\begin{tabular}{|c|c|c|c|c|c|c|c|}
\hline \multirow[t]{2}{*}{ Espécie } & \multicolumn{3}{|c|}{ Elementos vasculares } & \multicolumn{4}{|l|}{ Raios } \\
\hline & $\begin{array}{l}\text { Comprimento } \\
(\mu \mathrm{m})\end{array}$ & Esp. parede $(\mu \mathrm{m})$ & $\begin{array}{l}\text { Diâmetro Pr-v } \\
\qquad(\mu \mathrm{m})\end{array}$ & $\begin{array}{l}\text { Altura mult } \\
\text { (nº cells) }\end{array}$ & Altura & dlt $(\mu \mathrm{m})$ & $\begin{array}{l}\text { Larg. } \\
\text { mult } \\
(\mu \mathrm{m})\end{array}$ \\
\hline E. benthamii & $374^{\mathrm{c}}$ & $9,3^{\mathrm{a}}$ & $8^{\mathrm{b}}$ & $10^{\mathrm{b}}$ & & & $7,6^{\mathrm{a}}$ \\
\hline E. dunnii & $412 b^{c}$ & $7,8^{\mathrm{ab}}$ & $9^{\mathrm{ab}}$ & $12^{\mathrm{ab}}$ & & & $6^{\mathrm{b}}$ \\
\hline E. grandis & $530^{\mathrm{a}}$ & $7^{\mathrm{b}}$ & $11^{\mathrm{a}}$ & $10^{\mathrm{ab}}$ & & & $6,2^{\mathrm{b}}$ \\
\hline E. saligna & $503^{\mathrm{ab}}$ & $8,4^{\mathrm{ab}}$ & $10^{\mathrm{ab}}$ & $13^{\mathrm{a}}$ & & & $5,5^{\mathrm{b}}$ \\
\hline \multirow[t]{2}{*}{ Espécie } & Parênquima axial & & & Fibras & & & \\
\hline & $\begin{array}{c}\text { Altura da série } \\
(\mu \mathrm{m})\end{array}$ & Largura da série & $(\mu \mathrm{m})$ & $\begin{array}{c}\text { Comprimento } \\
(\mu \mathrm{m})\end{array}$ & $\begin{array}{c}\text { Largura } \\
(\mu \mathrm{m})\end{array}$ & \multicolumn{2}{|c|}{ Esp. parede $(\mu \mathrm{m})$} \\
\hline E. benthamii & $283^{c}$ & $17^{\mathrm{ab}}$ & & $903^{c}$ & $16^{\mathrm{b}}$ & \multicolumn{2}{|c|}{$3,4^{\mathrm{cb}}$} \\
\hline E. dunnii & $526^{a}$ & $15^{\mathrm{b}}$ & & $982^{\mathrm{b}}$ & $17^{\mathrm{ab}}$ & \multicolumn{2}{|c|}{$4,3^{\mathrm{a}}$} \\
\hline E. grandis & $485^{\mathrm{ab}}$ & $18,9^{\mathrm{a}}$ & & $1036^{\mathrm{ab}}$ & $18^{\mathrm{a}}$ & \multicolumn{2}{|c|}{$3,8^{\mathrm{b}}$} \\
\hline E. saligna & $410^{\mathrm{b}}$ & $18,2^{\mathrm{a}}$ & & $1078^{\mathrm{a}}$ & $15,7^{\mathrm{b}}$ & \multicolumn{2}{|c|}{$3,2^{\mathrm{c}}$} \\
\hline
\end{tabular}

Onde: Esp. Parede: Espessura da parede; Diâmetro Pr-v: Diâmetro das pontoações raio-vasculares; Altura mult: Altura de raios multisseriados; Larg. mult: Largura de raios multisseriados; Esp. Parede: espessura da parede; $\mu$ m: micrômetros. ${ }^{* *}$ Médias seguidas pela mesma letra na coluna não diferem entre si pelo Teste de Tukey à $5 \%$ de significância.

Where: Esp. Wall: Wall thickness; Diameter Pr-v: Diameter of the radio-vascular streaks; Height mult: Height of multi-serrated rays; Larg. Mult: Width of multi-serrated rays; Esp. Wall: wall thickness; Mm: micrometers. ${ }^{\star *}$ Means followed by the same letter in the column do not differ by Tukey's test at $5 \%$ significance. 


\section{Variação radial das dimensões das fibras da}

\section{madeira}

Para as quatro espécies verificou-se aumento no comprimento das fibras no sentido do cerne para o alburno (Figura 3). Essa tendência de variação tem sido exaustivamente relatada para diferentes espécies de eucalipto por Tomazello Filho (1985a, 1985b, 1985c, 1987) bem como para a maioria das espécies florestais (BUTTERFIELD et al., 1993; ADAMOPOULOS; VOUGARIDIS, 2002; HONJO et al., 2005; QUILHÓ et al., 2006, SUCKOW et al., 2009, LIMA et al., 2011 e CARRILLO et al. 2015).

O aumento do comprimento das fibras, conforme a árvore aumenta sua idade, é resultado do acréscimo no comprimento das células que as originam, denominadas fusiformes iniciais. A estabilização para inúmeras espécies de eucalipto, somente irá ocorrer quando as células do câmbio atingirem comprimento máximo, iniciando a formação da madeira caracterizada como adulta (TOMAZELLO FILHO, 1987).

Fibras com o menor comprimento médio, nas posições cerne, cerne periférico e alburno, foram verificadas em $\mathrm{Eu}$ calyptus benthamii, respectivamente 647, 801 e $903 \mu \mathrm{m}$. Embora menores que as demais, esses resultados podem ser considerados bons para fabricação de polpa branqueada, segundo recomendação de Foelkel (2007).

Quanto à largura total das fibras (Figura 3), Eucalyptus dunnii, E. grandis e E. saligna mostraram tendência semelhante, com ligeiro aumento na posição cerne para cerne periférica e, sequencialmente, uma pequena redução dos va-
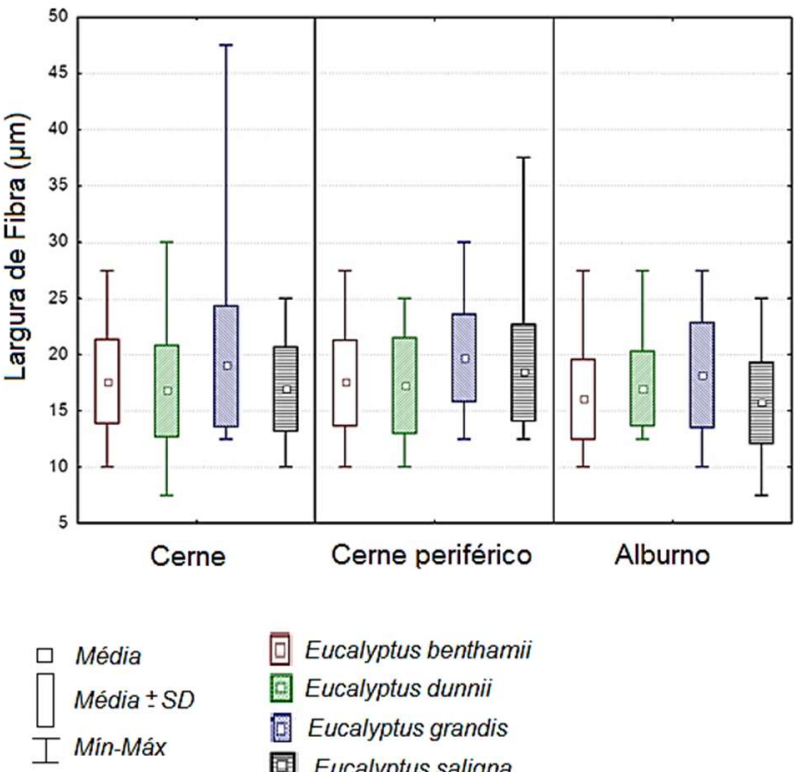

$$
\begin{aligned}
& \text { [ Eucalyptus benthamii } \\
& \text { 1. Eucalyptus dunnii } \\
& \text { [0. Eucalyptus grandis } \\
& \text { 圆 Eucalyptus saligna }
\end{aligned}
$$
periférico e alburno.

Figure 3. Radial variation of the length, width and wall thickness of the fibers, evaluated at the core, peripheral core and sapwood positions. 
lores no alburno. Já em E. benthamii, fibras mais largas são encontradas no cerne e decrescem até o alburno.

Padrão de variação semelhante foi determinado por Pirralho et al. (2014), em Eucalyptus camaldulensis, E. maculata, E. melliodora e E. sideroxylon, com um ligeiro aumento até a posição cerne periférico e posterior diminuição na largura total do tecido fibroso. Contrariamente, Tomazello Filho (1987) aponta que em E. globulus, E. pellita e E. acmenioides o parâmetro largura das fibras tende a aumentar no sentido medulacasca.

Para a espessura da parede das fibras não é possível definir uma tendência geral de variação (Figura 3). Eucalyptus benthamii e E. grandis apresentam aumento entre o cerne e o cerne periférico e, então, redução da espessura celular. Em $E$. dunnii e E. saligna verificou-se gradual aumento no sentido cerne para alburno.

Sobre a espessura da parede das fibras, há de se salientar a ausência de um padrão definido entre as inúmeras espécies de Eucalyptus, posto que, Brasil e Ferreira (1972) encontraram um aumento radial em E. grandis e Tomazello Filho (1987) em E. globulus, E. pellita e E. acmenioides, enquanto Sharma et al. (2005) verificaram decréscimo radial para as fibras de $E$. tereticornis, E. propinqua e E. sideroxylon.
Verifica-se que até os 5 anos, idade de observação das madeiras em estudo, não houve tendência de estabilização dos valores de comprimento, largura e espessura das fibras, podendo-se obter ganhos adicionais em qualidade, mantendose as árvores no campo por períodos maiores. Esse padrão de variação de fibras indica que a camada cambial dos eucaliptos ainda encontra-se formando madeira caracterizada como juvenil.

\section{Índices de qualidade da madeira para celulose}

\section{e papel}

Os índices qualitativos dos quatro eucaliptos (Tabela 3), determinados a partir da morfologia das fibras, fornecem um diagnóstico prévio do potencial dessas espécies para produção de celulose e papel. Para o índice de Runkel, o fator de Luce e a fração parede, Eucalyptus dunnii apresenta os maiores valores, por conter fibras de paredes mais espessas, como anteriormente mencionado na sua descrição quantitativa (Tabela 2).

O índice de Runkel, assim como a fração parede, relacionam-se à firmeza da fibra, sendo que, quanto maior o seu valor, mais rígida essa pode se tornar, afetando as propriedades de resistência do papel (GONÇALEZ et al., 2014).

Tabela 3. Índices indicativos da qualidade da madeira para produção de celulose e papel.

Table 3. Indices indicative of wood quality for pulp and paper production.

\begin{tabular}{|c|c|c|c|c|c|}
\hline Espécies & índice de Runkel & fração de parede (\%) & flexibilidade & rigidez & fator de Luce \\
\hline \multirow[t]{2}{*}{ E. benthamii } & $0,77 \pm 0,21$ & $42,61 \pm 7,12$ & $0,57 \pm 0,07$ & $57,39 \pm 8,72$ & $0,50 \pm 0,09$ \\
\hline & $(27,72)$ & $(16,71)$ & $(12,41)$ & $(15,19)$ & $(18,12)$ \\
\hline \multirow[t]{2}{*}{ E. dunnii } & $1,07 \pm 0,28$ & $51,53 \pm 8,08$ & $0,50 \pm 0,07$ & $58,96 \pm 11,09$ & $0,60 \pm 0,09$ \\
\hline & $(25,95)$ & $(15,62)$ & $(13,81)$ & $(18,81)$ & $(14,46)$ \\
\hline \multirow[t]{2}{*}{ E. grandis } & $0,76 \pm 0,25$ & $41,99 \pm 7,90$ & $0,58 \pm 0,08$ & $58,00 \pm 9,18$ & $0,50 \pm 0,10$ \\
\hline & $(32,83)$ & $(18,81)$ & $(13,62)$ & $(15,82)$ & $(20,40)$ \\
\hline \multirow[t]{2}{*}{ E. saligna } & $0,72 \pm 0,19$ & $41,11 \pm 6,06$ & $0,59 \pm 0,06$ & $68,96 \pm 7,52$ & $0,49 \pm 0,08$ \\
\hline & $(25,78)$ & $(14,73)$ & $(10,28)$ & $(10,91)$ & $(16,06)$ \\
\hline
\end{tabular}

Onde: valor médio \pm desvio padrão (coeficiente de variação).

Where: mean value \pm standard deviation (coefficient of variation). 
Barrichelo e Brito (1976) propõe cinco grupos para o índice de Runkel: as fibras classificadas no grupo I (até 0,25 ) são consideradas excelente para papel, do grupo II $(0,25-0,5)$ muito boas, no grupo III $(0,5-1,0)$ boas, no grupo IV $(1,0$ 2,0 ) regulares e no grupo $\mathrm{V}$ (acima de 2,0) não devem ser usadas para produção de papel tendo em vista o baixo grau de colapso. Segundo Foelkel et al (1978) celulose com fibras cujo índice seja superior a 1,5 são desaconselháveis para a indústria, sendo considerada de boa qualidade a que apresentar índice inferior a 1. Sob esse aspecto, Eucalyptus benthamii, E. grandis e E. saligna, podem ser classificadas como boas para produção de papel, enquanto E. dunnii é considerada regular.

Para a fração parede celular, todas as espécies encontramse dentro do limite de até $60 \%$ recomendado por Foelkel et al. (1978). Na prática, as indústrias admitem que quando a fração parede for maior que $40 \%$, as fibras serão mais rígidas e difíceis de se colapsarem e, assim, produzem um papel de rede mais solta e frouxa, sem muita ligação entre fibras. Como resultado final, os papéis correspondentes são mais porosos, volumosos, rugosos e absorventes (FOELKEL, 2007).

Ainda conforme o autor, polpas produzidas com espécies de paredes finas, como E. saligna, resultam em maior população fibrosa, maior entrelaçamento de fibras e possibilidades de contatos fibra a fibra. Com isso, melhoram as propriedades de tração, estouro, rasgo, dobramento, resistência superficial e resistência interna da folha.

A respeito do coeficiente de flexibilidade, o maior valor foi obtido para E. saligna $(0,59)$, que apresenta, dentre os demais eucaliptos, fibras mais longas e menos espessas. O coeficiente de flexibilidade, remete a facilidade da união entre as fibras, e, segundo Bektas et al. (1999) valores entre 0,50 - 0,75, como encontrados no estudo, classificam as fibras como flexíveis, que, ao se entrelaçarem, tendem a formar um papel altamente resistente. Conforme Saikia et al. (1997), espécies com bons valores de flexibilidade, caso de E. saligna, são adequadas para produção de papéis com alta resistência mecânica, tais como os de escrita, impressão, embalagem.

Por outro lado, a presença de fibras curtas, como evidenciado em E. benthamii, refletem em baixos valores de rigidez, que por sua vez, reduz drasticamente a resistência do papel produzido, face, primordialmente, a esse tipo de fibras possuir baixa superfície de contato fibra-fibra e acarretar em deficiência na colagem.

No estudo evidencia-se relação direta entre os coeficientes de flexibilidade e de rigidez com o comprimento das fibras. Ao passo que E. saligna, com fibras longas $(1078 \mu \mathrm{m})$ apresenta maiores valores para os coeficientes $(0,59$ de flexibilidade e 68,96 de rigidez); em E. benthamii as fibras de menor comprimento $(903 \mu \mathrm{m})$, resultam em coeficientes com valores mais baixos, 0,57 para flexibilidade e 57,39 para rigidez.

O fator de luce aparece com valores médios variando de 0,60 em E. dunnii, seguido por E. benthamii e E. grandis $(0,50)$ e E. saligna $(0,49)$. Relacionado com a densidade final da folha de papel, o fator de luce, pode ser uma propriedade utilizada na seleção dos exemplares quanto à qualidade da madeira.

Quanto ao gênero Eucalyptus a literatura é farta de trabalhos que retratam índices qualitativos. Pirralho et al. (2014) menciona para E. camaldulensis, respectivamente, 0,79 para o índice de Runkel, $43,5 \%$ para a proporção de parede, 0,56 para o coeficiente de flexibilidade e 0,51 para o fator de Luce; altamente semelhantes aos valores encontrados para o $E$. benthamii presentemente investigado. Ferreira et al. (2013), relatou um índice de Rukel de 0,45 para celulose comercial de E. globulus. Ona et al. (2001) avaliando E. camaldulensis e E. globulus mencionam, respectivos valores para Runkel, 0,42 e 0,39 e para coeficiente de flexibilidade 0,69 e 0,73. Contudo, cabe mencionar que para uma comparação fiável com os índices 
de qualidade mencionados na literatura, as espécies devem, pelo menos, possuir idades semelhantes.

\section{Conclusões}

Poros agrupados em três ou mais unidades identificam Eucalyptus saligna, assim como a presença de parênquima axial paratraqueal aliforme em E. dunnii corrobora na diferenciação dessa espécie. A anatomia quantitativa auxilia na distinção entre as quatro espécies pesquisadas.

A presença de traqueídes vasculares em Eucalyptus benthamii aparece como novidade e, até o presente momento, não foi mencionada em estudos anatômicos da espécie.

Foi observada variação radial nas características das fibras nas quatro espécies em estudo. Não houve tendência de estabilização do crescimento dos tecidos nas posições cerne, cerne periférico e alburno, o que caracteriza a madeira como juvenil e recomenda manter as árvores no campo por um período maior.

Os índices de qualidade da madeira mostram que $E \mathbf{E}$ calyptus benthamii, E. grandis e E. saligna, podem ser classificadas como boas para produção de papéis com alta resistência mecânica, tais como os de escrita, impressão, embalagem, enquanto E. dunnii pode ser indicada para papéis do tipo absorventes. A morfologia das fibras de E. benthamii, espécie ainda incipiente na produção de celulose, demonstra seu potencial para tal finalidade.

\section{Agradecimentos}

Os autores agradecem à empresa CMPC - Celuse Riograndense pela doação do material de estudo.

\section{Referências}

ADAMOPOULOS, S; VOULGARIDIS E. Within-tree variation in growth rate and cell dimensions in the wood of black locust (Robinia pseudoacacia L.). IAWA Jornal., v.23, n. 2, p. 191-199, 2002. DOI: 10.1163/22941932-90000297

ALVES, I. C.; GOMIDE, J. L.;COLODETTE, J. L.;SILVA, E. D. Caracterização tecnológica da madeira de Eucalyptus benthamii para produção de celulose kraft. Ciência Florestal, Santa Maria, v. 21, n. 1, p. 167-174, jan.-mar., 2011. DOI: http://dx.doi.org/10.5902/198050982759

ALZETE, B. A. Estrutura anatômica da madeira de clones de Eucalyptus. Revista investigaciones aplicadas., Medellín, v. 5, p. 1-14, 2009.

BARRICHELO, L. E. G.; BRITO, J. O. A madeira das espécies de eucalipto como matéria-prima para a indústria de celulose e papel. Série Divulgação PRODEPEF, Brasília, n. 13, p. 1$145,1976$.

BEKTAS, I.; TUTUS, A.; EROGLU, H. A study of the suitability of Calabrian Pine (Pinus burtia, Jen) for pulp and paper manufacture. Turkish Journal of Agriculture and Forestry, v. 23, n. 3, p. 589-597. 1999.

BRASIL, M. A. M.; FERREIRA, M. Variação da densidade básica e das característcas das fibras em Eucalyptus grandis Hill ex Maiden ao nível do DAP. IPEF, n.5, p.81-90, 1972.

BRISOLA, S. H.; DEMARCO. D. Análise anatômica do caule de Eucalyptus grandis, E. urophylla e E. grandis $\mathrm{x}$ urophylla: desenvolvimento da madeira e sua importância para a indústria. Scientia Forestalis, v. 39, n. 91, p. 317-330, set. 2011.

\section{BURGER, L. M.; RICHTER, H. G. Anatomia da Madeira.} São Paulo: Ed. Nobel. 1991. 154 p.

BUTTERFIELD, R. P.; CROOK, R. P.; ADANS, R.; MORRIS, R. Radial variation in wood specific gravity, fibre length and vessel area for two Central American hardwoods: Hyeronima alchorneoides and Vochysia guatemalensis: natural and plantation - grown trees. IAWA Journal., v. 14, p. 153-161. 1993. DOI: $10.1163 / 22941932-90001310$

CARRILLO, I.; AGUAYO, M. G.; VALENZUELA, S.; MENDONÇA, R. T. Variations in wood anatomy and fiber biometry of Eucalyptus globulus genotypes with different wood density. Wood Research., v. 60, n. 1, p. 1-10, 2015.

DADSWELL, H. E.; BURNELL, M. Methods for the identification of the coloured woods of the genus Eucalyptus. Council for Scientific and Industrial Research, Melbourne. Division of Forest Products Technical Paper 5. 1932. 
DÉTIENNE, P.; JACQUET, P. Atlas d'identification des bois de l'Amazonie et des regions voisines. Centre Technique Forestier Tropical, Nogent-sur-Marne, 1983. 640 p.

FERREIRA, P. J. T., GAMELAS, J. A. F., CARVALHO, M. G. V. S., DUARTE, G. V., CANHOTO, J. M. P. L., PAS-SAS, R., Evaluation of the papermaking potential of Ailanthus altissima. Industrial Crops and Products, v. 42, n. 1, p. 538-542. 2013. DOI: http://dx.doi.org/10.1016/j.indcrop.2012.06.030.

FREUND, H. Handbuch der Mikroskopie in der Technik. Frankfurt: Umsham Verlag, 1970.

FOELKEL, C. B.; BARRICHELO, L. E. G. Tecnologia de celulose e papel. Piracicaba: ESALQ/USP, ESALQ, 1975. 207 p.

FOELKEL, C. E. B.; ZVINAKEVICIUS, C.; ANDRADE, J.; MEDEIROS, S. J. Eucaliptos tropicais na produção de celulose kraft. Belo Oriente, Cenibra, n. 68, 1978, 31 p.

FOELKEL, C. As fibras dos eucaliptos e as qualidades requeridas na celulose kraft para a fabricação de papel. In: $E u$ calyptus Online Book \& Newsletter, 2007. 48 p.

GOMIDE, J. L.; COLODETTE, J. L.; OLIVEIRA, R. C.; SILVA, C. M. Caracterização tecnológica, para produção de celulose, da nova geração de clones de Eucalyptus do Brasil. Revista Árvore, Viçosa, v. 29, n. 1, p. 129-137, 2005. DOI: http://dx.doi.org/10.1590/S0100-67622005000100014

GONÇALEZ, J. C.; SANTOS, G. L.; SILVA JUNIOR, F. G.; MARTINS, I. S.; COSTA, J. A. Relações entre dimensões de fibras e de densidade da madeira ao longo do tronco de $E u$ calyptus urograndis., Scientia Forestalis, v. 42, n. 101, p. 8189, mar.; 2014.

GONÇALVES, T. A. P.; BALLARIN, A. W.; NISGOSKI, S.; MUNIIZ, G. I. B. A contribution to the identification of charcoal origin in Brazil I - anatomical characterization of Corymbia and Eucalyptus. Maderas. Ciencia y tecnologia., v. 16, n. 3, p. 323-336, 2014. DOI:10.4067/S0718-221X2014005000025

HONJO, K.; FURUKAWA, I.; SAHRI, M. H. Radial variation of fiber length increment in Acacia mangium Leinden. IAWA Journal., v. 26, n. 3, p. 339-352, 2005.

IAWA COMMITTEE. IAWA list of microscopic features for hardwood identificacion. IAWA Journal., v. 10, n. 3, p. 218 359, 1989.

INDÚSTRIA BRASILEIRA DE ÁRVORES. Relatório IBÁ 2016. São Paulo, 2016. 100 p.
LIMA, I. L.; LOUGUI, E. L.; GARCIA, M. F.; ZANATTO, A. C. S.; FREITAS, M. L. M.; FLORSHEIM, S. M. B. Variação radial da densidade básica e dimensões celulares da madeira de Cariniana legalis (Mart.) O. Kuntze em função da procedência. Cerne., v. 17, n. 4, p. 517-524, 2011.

LUCE, G. E. The physics and chemistry of wood pulp fibers. In: STAP, n. 8, TAPPI, New York, 1970, p. 278.

MARCHIORI, J. N. C. Estudo anatômico do xilema secundário e da casca de Acacia recurve Benth. (Leguminosae Mimosoideae). Ciência e Natura, Santa Maria, n. 4, p. 95-105, 1982.

METCALFE, C. R.; CHALK, L. Anatomy of the dicotyledons. Wood structure and conclusions of the general introdution. Oxford: Clarendon Press, 1983.

MILANEZ, A.C.; FOELKEL, C.E.B. Processos de deslignificação com oxigênio para a produção de celulose de eucalipto. In: Congresso anual da ABCP, 14., São Paulo. Anais... São Paulo: ABTCP. p. 37-110, 1981.

NISGOSKI, S.; MUÑIZ, G. I. B.; KLOCK, U. Caracterização anatômica da madeira de Eucalyptus benthamii Maiden et Cambage. Ciência Florestal, Santa Maria, v. 8, n. 1, p. 67-76. 1998.

OGBONNAYA, C. I.; ROY-MACAULEY, H.; NWALOZIE, M. C.; ANNEROSE, D. J. M. Physical and histochemical properties of kenaf (Hibiscus cannabinus L.) grown under water deficit on a sandy soil. Industrial Crops and Products, v. 7, p. 9-18, 1997. DOI: http://dx.doi.org/10.1016/S09266690(97)00034-4

OLIVEIRA, J. G. L.; OLIVEIRA, J. T. S.; ABAD, J. I. M.; SILVA, A. G.; FIEDLER, N. C.; VIDAURE, G. B. Parâmetros quantitativos da anatomia da madeira de Eucalipto que cresceu em diferentes locais. Revista Árvore, Viçosa-MG, v. 36, n. 3, p. 559-567, 2012. DOI: http://dx.doi.org/10.1590/S010067622012000300018

ONA, T.; SONODA, T.; ITO, K.; SHIBATA, M.; TAMAI, Y.; KOJIMA, Y.; OHSHIMA, J.; YOKOTA, S.; YOSHIZAWA, N. Investigation of relationships between cell and pulp properties in Eucalyptus by examination of within-tree property variations. Wood Science and Technology, v. 35, n. 3, p. 229243, 2001.DOI: http://dx.doi.org/10.1007/s002260100090

PIRRALHO, M.; FLORES, D.; SOUSA, V. B.; QUILHÓ, T.; KNAPIC, S.; PEREIRA, H. Evaluation on paper making potential of nine Eucalyptus species based on wood anatomical 
features. Industrial Crops and Products, v. 54, p. 327-334 2014. DOI: http://dx.doi.org/10.1016/j.indcrop.2014.01.040

QUILHÓ, T.; MIRANDA, I.; PEREIRA, H. Within-tree variation in wood fibre biometry and basic density of the urograndis eucalypt hybrid (Eucalyptus grandis $\mathrm{E}$. urophylla). IAWA Journal., v. 27, p. 243-254, 2006. DOI: 10.1163/22941932-90000152

RAYMOND, C. A.; MUNERI, A. Nondestructive sampling of Eucalytpus globulus and E.nitens for wood properties. I. Basic density. Wood Science and Technology, v. 35, n. 1, p. 27-39, 2001. DOI: doi:10.1007/s002260000078

RECORD, S. J.; HESS R.W. Timbers of the New World. New Haven: Yale University Press, 1949. 640 p.

REIS, C. A. F., GONÇALVES, F. M. A., RAMALHO, M. A. P., ROSADO, A. M. Estratégias na seleção simultânea de vários caracteres no melhoramento do Eucalyptus. Ciência

Florestal. v.25, n.2, Santa Maria, 2015. DOI:

http://dx.doi.org/10.5902/1980509818465

RUNKEL, R.O.H. PULP FROM TROPICAL WOOD. TAAP., v. 35, n 4, p. 174-178, 1952.

SAIKIA, S. N.; GOSWAMI, T.; ALI, F. Evaluation of pulp and paper making characteristics of certain fast growing plants. Wood Science and Technology, v. 31, p. 467-475, 1997. DOI:10.1007/BF00702569

SANTOS, S. R. dos.; SIEGLOCH, A. M.; MARCHIORI, J. N. C. Análise de agrupamento de 16 gêneros e 71 espécies de Myrteae, com base em dados da anatomia da madeira. Balduinia., Santa Maria, n. 47, p. 24-33, 2015. DOI: http://dx.doi.org/10.5902/2358198017231

SHARMA, S. K.; RAO, R. V.; SHUKLA, S. R.; KUMAR, P.; SUDHEENDRA, R.; SUJATHA, M.; DUBEY, Y. M. Wood quality of coppiced Eucalyptus tereticornis for value addition. IAWA Journal., v. 26, n. 1, p. 137-147, 2005. DOI: $10.1163 / 22941932-90001608$

SILVA, L. D.; HIGA, A. R.; SANTOS, G. A. Silvicultura e melhoramento genético de Eucalyptus benthamii. Curitiba: FUPEF, 2012. $150 \mathrm{p}$.

SUCKOW, I. M. S.; LONGUI, E. L.; LIMA, I. L.; FLORSHEIM, S. M. B.; AGUIAR, O. T. Anatomia da madeira e densidade básica de angico-branco Anadenanthera colubrina (Vell.) Brenan. Instituto Florestal Série Registros., v. 40, p. 131-135, 2009.
TOMAZELLO FILHO, M. Estrutura anatômica da madeira de oito espécies de eucaliptos cultivadas no Brasil. IPEF, Piracicaba, v. 29, p. 25-36, 1985a.

TOMAZELLO FILHO, M. Variação radial da densidade básica e da estrutura anatômica da madeira do Eucalyptus saligna e E. grandis. IPEF, Piracicaba, v. 29, 37-45, 1985b.

TOMAZELLO FILHO, M. Variação radial da densidade básica e da estrutura anatômica da madeira do Eucalyptus gummifera, E. microcorys e E. pilularis. IPEF, Piracicaba, v. 30, p. 45-54, 1985c.

TOMAZELLO FILHO M. Variação da densidade básica e da estrutura anatômica da madeira de Eucalyptus globulus, Eucalyptus pellita e Eucalyptus acmeniodes. IPEF.,v. 36, p. 3542, 1987.

VLIET, G. J. C. van; BAAS, P. Wood anatomy and classification of the Myrtales. Annals of the Missouri Botanical Garden, n. 71, p. 783-800, 1984.

WIEDENHOEF, A. C.; MILLER, R. B. Structure and function of wood. I landbook of wood chemistry and wood composites. CRC Press-Taylor \& Francis Group. 6000 Broken Sound Parkway NW, STE 300. Boca Raton. FL 33487-2742 USA. p. 9-33, 2005.

WILKES, J. Variations inwoodanatomy within species of $E u$ calyptus. IAWA Journal., v. 9, n. 1, p. 13-23, 1988. 